\title{
Pseudo Tumoral Form of Mediastinal Tuberculosis: Case Report
}

\author{
F. Z. Abakka ${ }^{1 *}$, I. Zouita ${ }^{1}$, Y. Zouine ${ }^{1}$, D. Basraoui ${ }^{1}$, H. Jalal ${ }^{1}$
}

${ }^{1}$ Radiology Mother Child Department, CHU Mohammed VI, University Cadi Ayad, Marrakech, Morocco

DOI: $\underline{10.36347 / \text { simcr.2021.v09i04.042 }}$

| Received: 17.03.2021 | Accepted: 26.04.2021 | Published: 30.04.2021

*Corresponding author: F. Z. Abakka

Abstract

Case Report

Mediastinal pseudotumoral tuberculosis is a rare form of Mycobacterium tuberculosis infection, characterized by marked diagnostic difficulty due to the absence of specific clinical or biological signs and deceptive imaging mimicking a neoplastic pathology. In this article, we report a case of mediastinal pseudotumoral tuberculosis that was treated with a thoracic CT scan and a dorsal MRI scan, which showed a posterior mediastinal lesion process infiltrating the spinal canal and the dorsal soft tissues and suggesting a tumoral or infectious origin. The tubercular origin was retained in the face of presumptive arguments and the patient was treated as lymph node tuberculosis and put on anti-bacillary drugs based on streptomycin, rifampicin, isoniazid and pyrazinamide according to the $2 \mathrm{RHZ} / 4 \mathrm{RH}$ protocol with a very good clinical and paraclinical evolution.

Keywords: Mediastinal tuberculosis, biological signs, CT scan, rifampicin.

Copyright $(\mathcal{C} 2021$ The Author(s): This is an open-access article distributed under the terms of the Creative Commons Attribution 4.0 International License (CC BY-NC 4.0) which permits unrestricted use, distribution, and reproduction in any medium for non-commercial use provided the original author and source are credited.

\section{INTRODUCTION}

Tuberculosis is a public health problem [1] due to its widespread nature, particularly in developing countries. The mediastinal location is rare. Diagnosis is difficult due to the non-specific nature of its clinical signs and the absence of characteristic radiological findings. It can sometimes simulate a mediastinal tumor, which poses a diagnostic challenge for radiologists and clinicians [2].

\section{ObSERVATION}

We report the case of a 13 years old patient, without any particular pathological history, hospitalized in our training for chronic (more than 1 year) intense, insomniating and radiating inter scapular chest pain associated with a wet cough, a fever of $39 \mathrm{C}$ and night sweats.
The clinical examination revealed a stature weight delay (-2DS), a dorsal spinal syndrome with a painful fixed swelling of right paravertebral tissue consistency, pain on palpation of the rib cage and scapula and slight intercostal traction.

A BK assessment was performed: three sputum BKs came back negative, the expert gene negative and Quantiferon negative.

The CT scan (Fig-1) was in favor of posterior locally infiltrating mediastinal posterior masses extended from D4 to D11 with intraductal extension. Further investigation by dorsal MRI (Fig-2) showed a pre- and latero-vertebral lesion process infiltrating the posterior mediastinum, the spinal canal and the dorsal soft tissues opposite, suggesting a tumor or infectious origin. 


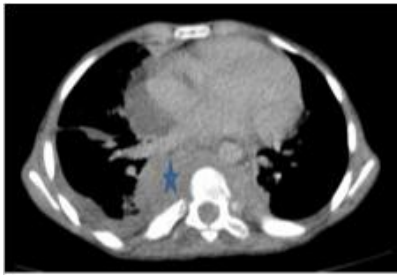

A
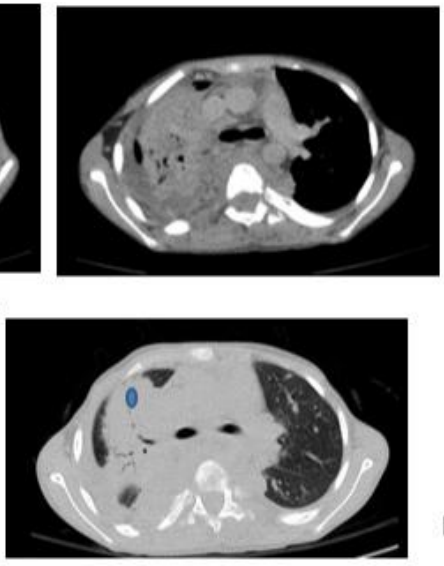

B
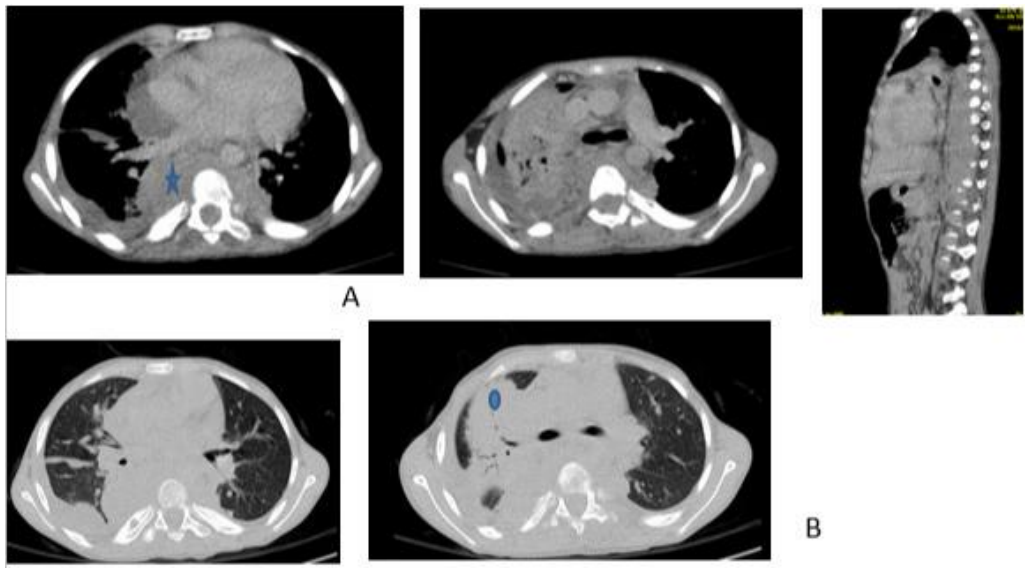

Fig-1: Thoracic CT scan with PDC injection, (A) mediastinal window and (B) parenchymal window: $₫$ Posterior locally infiltrating mediastinal masses extended from D4 to D11 with intraductal extension. Associated with a right apical akeolar condensation

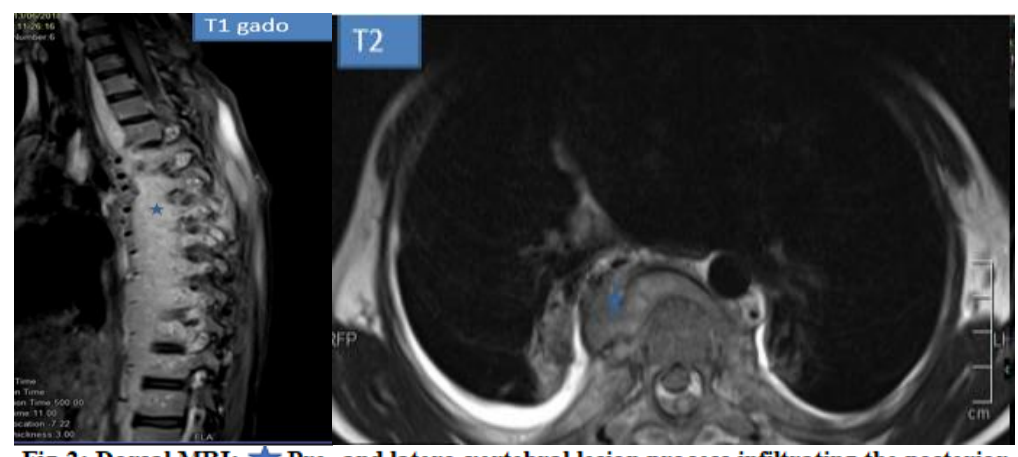

Fig-2: Dorsal MRI; 7 Pre- and latero-vertebral lesion process infiltrating the posterior mediastinum, spinal canal and facing dorsal soft tissues

Following the aggravation of his condition, a thoracic CT scan was performed 4 months later, revealing the persistence of the posterior mediastinal process with the appearance of a right thoracic basal necrotic mass and a right basal condensation site suggesting an infectious origin.
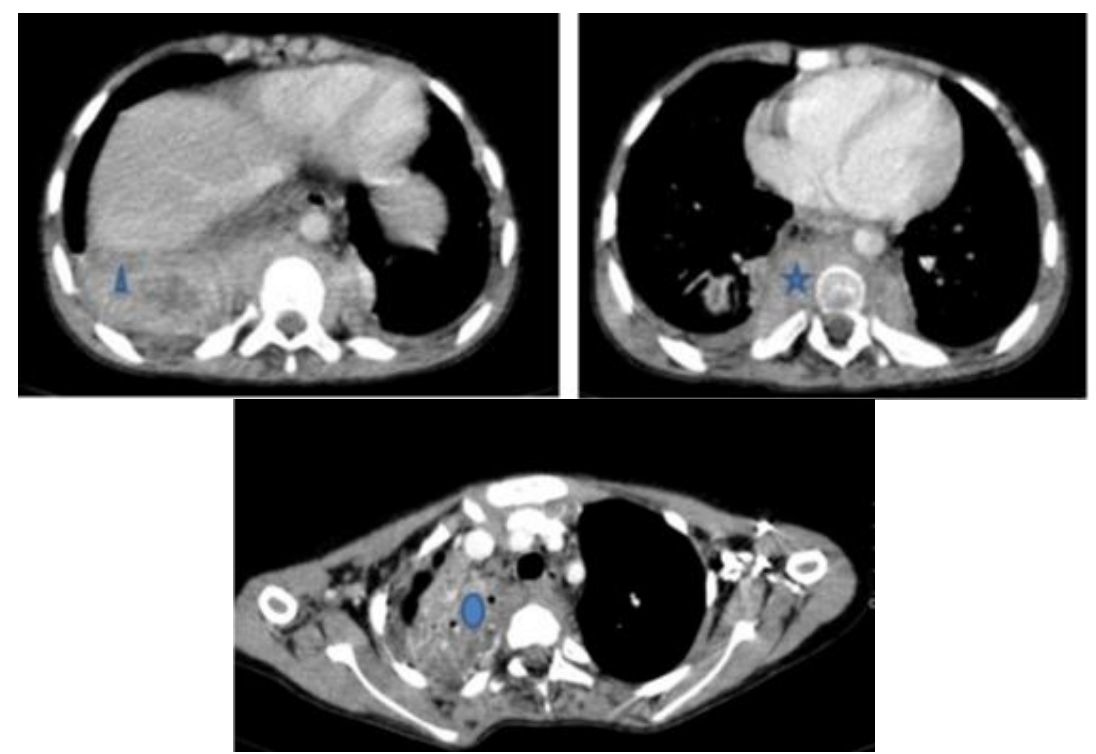

Fig-3: Thoracic CT scan (4 months later) with PDC injection, mediastinal window:

$\underset{\lambda}{ }$ Persistence of the locally infiltrating pre and latero vertebral lesional process.

Apical and postero-basal alveolar condensation.

$\triangle$ Right basithoracic necrotic mass. 
A biopsy of the parietal mass was performed with an anatomical pathological examination of an inflammatory macrophage granuloma in fibrosclerotic tissue.

The patient was treated for tuberculosis of the lymph nodes and placed on antibacterial drugs based on streptomycin, rifampicin, isoniazid and pyrazinamide according to the $2 \mathrm{RHZ} / 4 \mathrm{RH}$ protocol with a very good clinical and paraclinical evolution.

\section{DISCUSSION}

Mediastinal tuberculosis is a relatively common extra pulmonary form of tuberculosis, which is often difficult to diagnose [3].

Its prevalence is higher in developing countries than in developed countries. It is difficult to diagnose with certainty, and sometimes it is difficult to affirm the definitive cure of cases, hence the interest of multidisciplinary collaboration to improve the proportion of proven cases and better define the criteria for cure [4].

The majority of patients with lymph node tuberculosis are young and slightly predominantly female, which corresponds to our case $[5,6]$. It follows a bacillary dissemination with a pulmonary origin [7].

The pseudotumoral form of mediastinal tuberculosis is a rare form, like pulmonary tuberculosis it is characterized by an atypical and misleading radioclinical picture, which makes diagnosis difficult and delayed in most cases [8]. The clinical symptomatology is most frequently non-specific and not very evocative, in the form of cough, chest pain, and alteration of the general condition; these signs may point more towards a neoplastic pathology than an infectious one.

The diagnosis of GMTs is difficult to establish because of the profound nature of the masses, which poses the problem of etiological diagnosis, especially with lymphoma, sarcoidosis, lymph node metastases and neurogenic tumors [9]. This was the case for our patient because of the posterior mediastinal localization and the infiltrating character, lymphoma or nerve tumors, especially schwannomas, were evoked.

Acteriological samples are negative on direct examination and rarely positive on culture because of the solid and poorly oxygenated character of caseous lesions in pseudotumoral tuberculosis [10].

It is noted that the difficulty in diagnosing the pseudotumoral form of mediastinal tuberculosis is due to the non-specific clinical signs, the negativity of bacteriological samples, and the misleading aspects of the lesions on CT scans; nevertheless, imaging remains crucial in the diagnosis of the disease thanks to its interventional component represented by scan-guided biopsy samples [11]. However, the diagnosis must be made in the face of any mediastinum-pulmonary lesion, even if it is tumor-like, especially in an evocative epidemiological and clinical context.

The treatment of mediastinal pseudotumor tuberculosis is often easy, the diagnosis can be made on the basis of presumptive arguments, it is based on antibacillary drugs with the usual dosages and durations, generally leading to a complete cure of the disease and a favorable clinical evolution, as in the case of our patient.

\section{CONCLUSION}

Mediastinal pseudotumoral tuberculosis is unusual and deserves to be better known because it can simulate cancer. The main risk is to misunderstand a pathology that is curable by a specific medical treatment.

The etiological diagnosis of mediastinal masses can be difficult but remains dominated by the tubercular origin in our context. This allows us to make the diagnosis on the basis of presumptive arguments without resorting to other invasive procedures [12].

Conflicts of Interest: The authors do not declare any conflict of interest.

\section{REFERENCES}

1. Lanoix JP, Douadi Y, Borel A, Andrejak C, El Samad Y, Ducroix JP, Schmit JL. Traitement de la tuberculose ganglionnaire des recommandations à la pratique. Médecine et maladies infectieuse. 2011;41:87-91.

2. Meryem E, Nabil Moatassim B, Ittimade N. Role of imaging test in the diagnosis of pseudotumoral form of pulmonary tuberculosis: about three cases. Service de Radiologie Centrale, CHU Ibn Sina, Rabat, Maroc Case report. Mar 2020.

3. Mazza-Stalder J, Nicod L, Janssens JP. La tuberculose extrapulmonaire. Revue des Maladies Respiratoires. 2012;29(4):566-578.

4. Tuberculose ganglionnaire: aspects épidémiologiques, diagnostiques et thérapeutiques, à propos de 357 cas Pan Afr Med J. 2014.

5. El Bied B, Afif H, Mokahli S, Aichane A, Bouayad Z, Bellekhal N. La tuberculose ganglionnaire: à propos de 240 cas. Rev Mal Respir. 2007;24:27.

6. Rebbat A, Aliouane A, Ait Bachir H. Tuberculose ganglionnaire à propos de 101 cas. Rev Mal Respir. 2013;30:A166.

7. Huchon G. Tuberculose: infection et maladie. EMC - Pneumologie. 2012;9(4):1-2

8. Snene H, Mansour AB, Toujani S, Salah NB, Mjid M, Ouahchi Y, Mehiri N, Beji M, Cherif J, Louzir B. La tuberculose pseudotumorale, un diagnostic difficile. Revue des maladies respiratoires. 2018 Mar 1;35(3):295-304. 
9. Adila F, Chiba F, Ziane F, Brahimi T, Benamara S, Zitouni A. La tuberculose ganglionnaire médiastinale dans le service de pneumologie de l'hôpital central de l'armée: aspects épidémiologiques, cliniques, paracliniques et thérapeutiques. Revue des Maladies Respiratoires. 2018 Jan 1;35:A174.

10. Ouarssani A, Atoini F, Reda R, Lhou FA, Rguibi MI. Pseudotumoral form of pulmonary tuberculosis and diagnosis difficulties: about a case. Pan Afr Med J. 2013;14:81.
11. Fiogbe A, Liistro G, Hoton D, Pieters $T$. Mycobacterium avium tumoral infection mimicking a lung adenocarcinoma: a potential diagnostic pitfall. Rev Pneumol Clin. 2016 Apr;72(2):147-51.

12. Salah EB, Berraies A, Abid H, Ammar J, Kotti A, Hamzaoui A. La tuberculose ganglionnaire médiastinale de l'enfant: à propos de six cas. Revue des Maladies Respiratoires. 2012 Jan 1;29:A128. 